\title{
"de Winter" electrocardiogram pattern in inferior leads in proximal right coronary artery occlusion
}

\section{Patrón de ECG de "de Winter" en derivaciones inferiores en oclusión RCA proximal}

\author{
Samad Ghaffari', Leili Pourafkari, and Nader D. Nader ${ }^{3 *}$ \\ ${ }^{1}$ Cardiovascular Research Center, Tabriz University of Medical Sciences, Tabriz, East Azerbaijan, Iran; ${ }^{2}$ Catholic Health System, University at \\ Buffalo, Buffalo, New York; ${ }^{3}$ Department of Anesthesiology, University at Buffalo, Buffalo, New York, USA
}

A 58-year-old smoker man presented with acute retrosternal chest pain of $2 \mathrm{~h}$ duration. Electrocardiogram (ECG) showed $2 \mathrm{~mm}$ upsloping ST-segment depression at the $\mathrm{J}$ point in inferior leads with positive $\mathrm{T}$ waves, $1.5 \mathrm{~mm}$ ST elevation in lead aVR and reciprocal ST depression in lead I, as well as $1 \mathrm{~mm}$ ST elevation in lead V1 and V2 (Fig. 1). Urgent coronary angiography showed complete thrombotic occlusion of proximal right coronary artery (Fig. 2). The lesion was successfully treated with evacuation of the thrombus and placing a drug-eluting stent. ECG after procedure showed new $Q$ waves in inferior leads (Fig. 3). Subsequent clinical course was uneventful.

\section{Discussion}

"de Winter" ECG pattern characterized by upsloping junctional ST-segment depression with positive and tall symmetrical T waves in precordial leads is considered ST elevation myocardial infarction (STEMI) equivalent and was first described in $2008^{1}$. This pattern accounts for $2 \%$ of anterior STEMI cases and it is associated with proximal occlusion of the left anterior descending artery. A similar pattern in inferior leads has rarely been described in literature ${ }^{2,3}$. In this patient, the ST elevation in V1-V2 is likely secondary to the right ventricular involvement. Other pathologies that manifest with ST

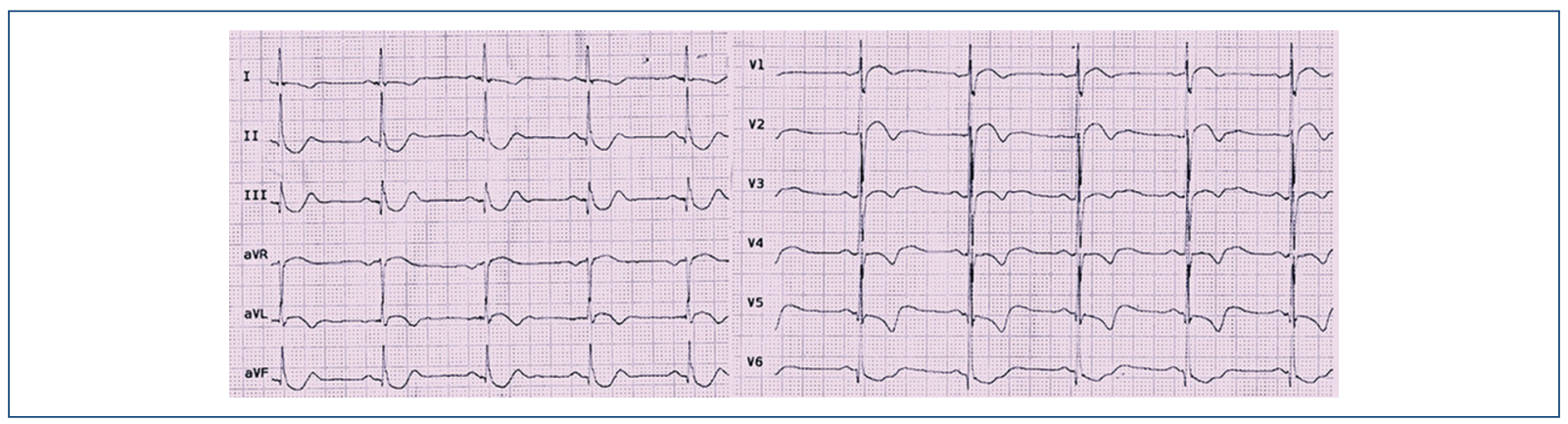

Figure 1. Electrocardiogram on presentations showing sinus rhythm, $2 \mathrm{~mm}$ upsloping ST-segment depression at the $\mathrm{J}$ point in inferior leads with positive T waves and $1.5 \mathrm{~mm}$ ST elevation in lead avR, and $1 \mathrm{~mm}$ ST elevation in V1 and V2 and $T$ inversion in anterior leads.

Correspondence:

*Nader D. Nader

E-mail: nnader@buffalo.edu
Date of reception: $24-06-2020$

Date of acceptance: 15-09-2020 DOI: 10.24875/ACME.M21000239
Available online: 02-07-2021 Arch Cardiol Mex (Eng). 2021;91(3):332-334 www.archivoscardiologia.com
article under the CC BY-NC-ND license (http://creativecommons.org/licenses/by-nc-nd/4.0/). 


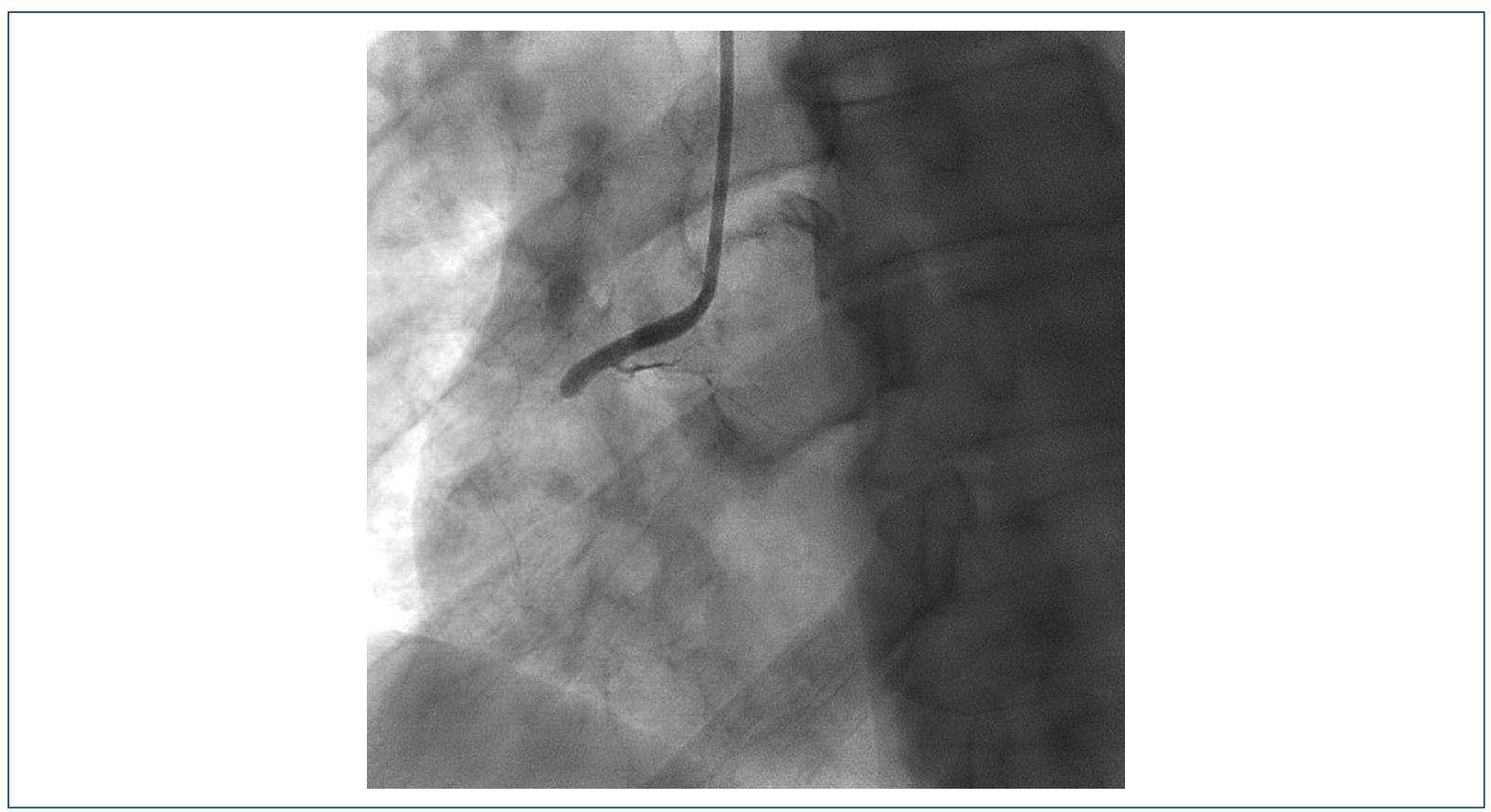

Figure 2. The right coronary angiogram in the left anterior oblique view showing the proximal occlusion of the right coronary artery.

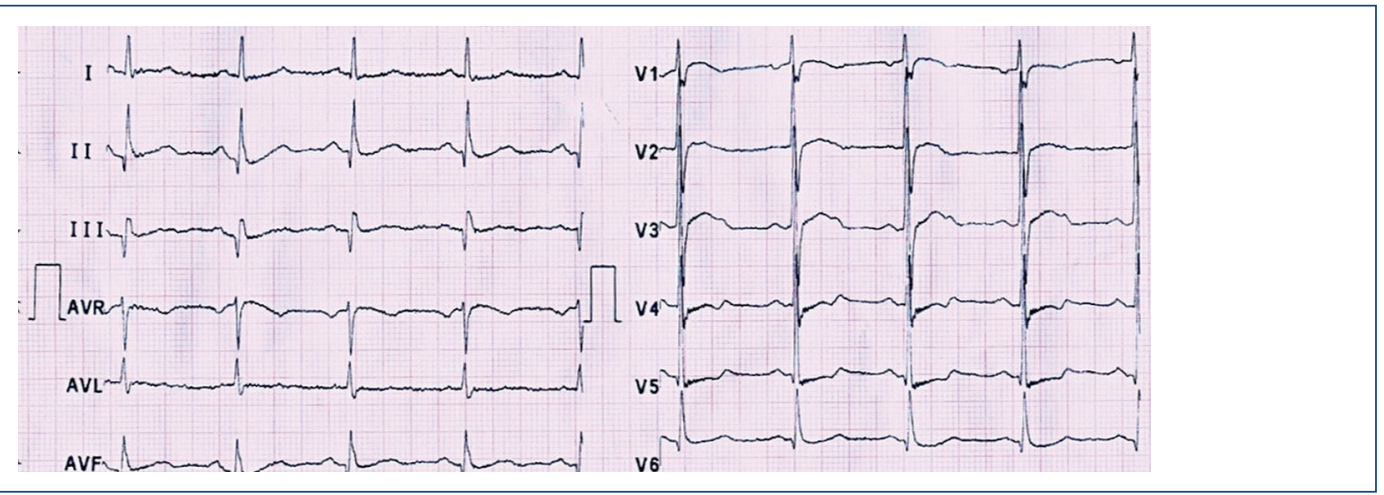

Figure 3. Electrocardiogram after the right coronary artery stenting showing new 0 waves and resolution of upsloping ST-segment depression of $\mathrm{J}$ point in inferior leads.

elevation in V1 and V2 include hyperkalemia, Brugada syndrome, hypercalcemia, class IC antiarrhythmic medications, and left ventricular hypertrophy ${ }^{4}$. It is essential to recognize of this variation of STEMI to avoid unnecessary delays in reperfusion therapies.

\section{Funding}

This research has not received any specific grant from public, commercial, or non-profit agencies.

\section{Conflicts of interest}

None.

\section{Ethical disclosures}

Protection of human and animal subjects. The authors declare that the procedures followed were in accordance with the regulations of the relevant clinical research ethics committee and with those of the Code 
of Ethics of the World Medical Association (Declaration of Helsinki).

Confidentiality of data. The authors declare that they have followed the protocols of their work center on the publication of patient data.

Right to privacy and informed consent. The authors have obtained the written informed consent of the patients or subjects mentioned in the article. The corresponding author is in possession of this document.

\section{References}

1. de Winter RJ, Verouden NJ, Wellens HJ, Wilde AA, Interventional Cardiology Group of the Academic Medical C. A new ECG sign of proximal LAD occlusion. N Engl J Med. 2008;359:2071-3.

2. Karna S, Chourasiya M, Chaudhari T, Bakrenia S, Patel U. De winter sign in inferior leads: a rare presentation. Heart Views. 2019;20:25-7.

3. Tsutsumi K, Tsukahara K. Is the diagnosis ST-segment elevation or Non-ST-segment elevation myocardial infarction? Circulation. 2018; 138:2715-7.

4. Wang K, Asinger RW, Marriott HJ. ST-segment elevation in conditions other than acute myocardial infarction. N Engl J Med. 2003; 349:2128-35 